Case Report

\title{
Guide Extension Catheter-Facilitated Reverse Controlled Antegrade and Retrograde Tracking for Retrograde Recanalization of Chronic Total Occlusion
}

\author{
Rohit Mody $\left(\mathbb{D},{ }^{1}\right.$ Debabrata Dash $\left(\mathbb{D},{ }^{2}\right.$ Bhavya Mody $\mathbb{D}^{3},{ }^{3}$ and Aditya Saholi $\mathbb{D}^{4}$ \\ ${ }^{1}$ Department of Cardiology, MAX Super Specialty Hospital, Bathinda, Punjab, India \\ ${ }^{2}$ Department of Cardiology, Zulekha Hospital, AL Zahra Street, Sharjah 457, UAE \\ ${ }^{3}$ Department of Medicine, Kasturba Medical College, Manipal, Karnataka, India \\ ${ }^{4}$ Department of Medicine, Irwin Hospital, Surja Ram Market, Malout, Punjab, India \\ Correspondence should be addressed to Rohit Mody; drmody_2k@yahoo.com
}

Received 23 November 2020; Revised 3 January 2021; Accepted 11 January 2021; Published 20 January 2021

Academic Editor: Yigit Canga

Copyright (c) 2021 Rohit Mody et al. This is an open access article distributed under the Creative Commons Attribution License, which permits unrestricted use, distribution, and reproduction in any medium, provided the original work is properly cited.

Background. In recent years, the retrograde approach has become a common practice in the treatment of chronic total occlusion (CTO) of coronary ostium which is arising abnormally and has an ambiguous proximal cap. In this case report, we report a case of retrograde percutaneous coronary intervention (PCI) done successfully on an abnormally originating artery which was guideliner assisted. Case Presentation. A 65-year-old gentleman with a history of hypertension, diabetes, and PCI presented to us with angina. Physical examination, electrocardiography (ECG), and echocardiography were done. Coronary angiography (CAG) revealed a normal left anterior descending artery (LAD), an anomalous circumflex (CX) artery arising from the right cusp. The abnormal CX had an implanted stent from which the abnormal right coronary artery (RCA) was arising and had a CTO. It also revealed the retrograde filling of distal RCA through grade 2 Werner collateral channels (CCs) from the LAD, a long CTO segment with a distal cap at the bifurcation. PCI of an RCA-CTO was scheduled utilizing a primary retrograde strategy, since antegrade ostium was abnormal in origin, and the patient was previously stented across the origin. The retrograde wire was externalized, and the procedure was completed with 3 overlapping drug-eluting stents (DESs). We used a guideliner which also assisted in the capture of retrograde corsair during the retrograde procedure of CTO [assisted reverse controlled antegrade and retrograde tracking (CART)]. These measures helped us to complete the CTO intervention successfully. Conclusion. The antegrade crossing is the most common approach to CTOs. However, it is sometimes difficult to penetrate the proximal hard ambiguous cap with guidewires, especially in the case of CTOs of anomalous coronary arteries because of a lack of support. Herein, we describe an iteration of reverse CART technique using a guide extensor catheter to facilitate externalizing the retrograde wire from false to true lumen.

\section{Introduction}

PCI of CTO has a lower success rate than PCI of nonoccluded coronary artery $[1,2]$. In 1990, the first retrograde approach technique was applied for CTO via a degenerated saphenous vein graft. Over the next 2 decades, septal collateral branches were considered to be novel access for the retrograde approach [3]. With the emergence of new equipment and important iterations, this approach has become safer, faster, and more successful [4]. In this case report, we report a case of retrograde
PCI done successfully on an abnormally originating artery which was guideliner assisted. Mozid et al. similarly described the utility of a guideliner catheter in retrograde PCI of a CTO with reverse CART - the "Capture" technique [5].

\section{Case Presentation}

2.1. History. A 65-year-old gentleman presented with Canadian Cardiovascular Society (CCS) Class III angina despite being on optimal medical therapy, including beta-blocker 


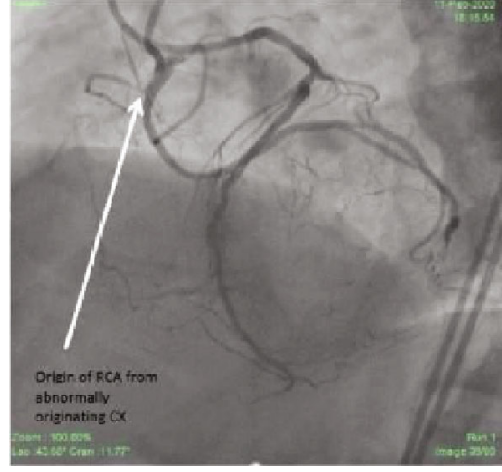

(a)

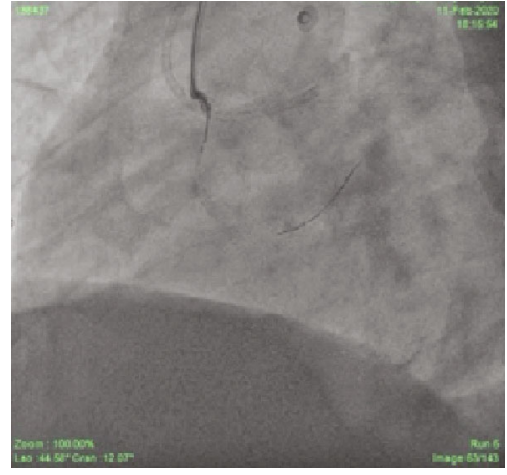

(b)

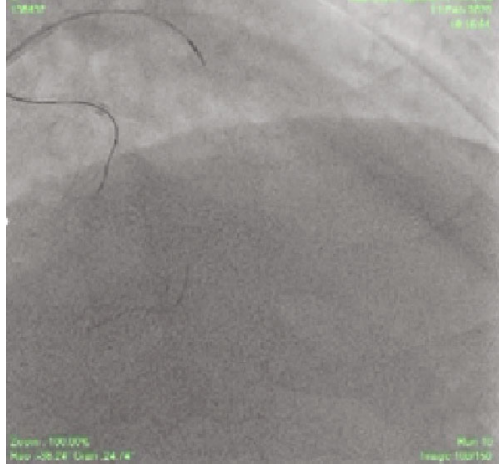

(c)

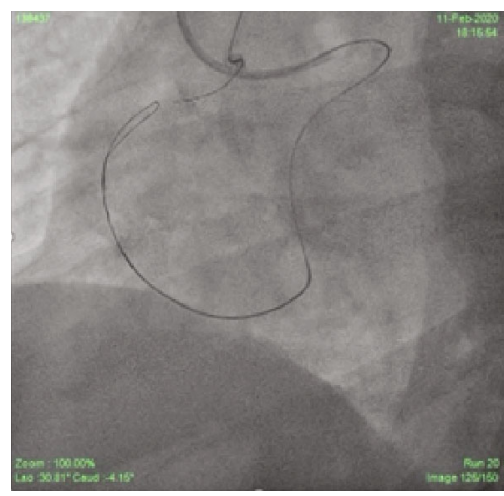

(d)

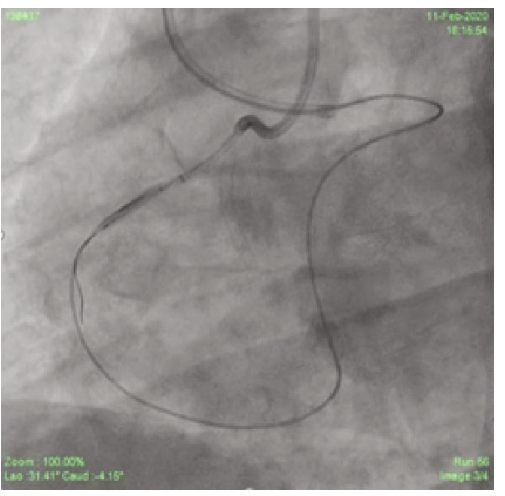

(e)

FIgure 1: (a) Retrogradely filling abnormal RCA originating from abnormally originating CX. (b) POT done in CX stent. (c) Sion blue and Corsair crossed retrogradely. (d) Knuckle pilot wire reaching proximal RCA subintimaly. (e) Guideliner assisted creation of common space antegradely.

(Metocard XL $100 \mathrm{mg}$ OD), long-acting nitrates (Nitrocontin $6.4 \mathrm{mg}$ BD), calcium channel blocker (Dilzem SR $120 \mathrm{mg}$ OD), and Ranolazine (Rancad $1 \mathrm{~g}$ OD). In addition to that, the patient used to take sublingual nitrates as and when required for angina. He was a known case of hypertension, diabetes, and a post-PCI of the proximal segment of an anomalous CX artery arising from the right cusp. The implanted stent was covering the abnormal origin of RCA from CX which had a CTO. He was also on dual antiplatelet therapy (DAPT) with aspirin $75 \mathrm{mg}$ OD and Clopidogrel $75 \mathrm{mg}$ OD as a post-PCI regimen. Also, the patient was on an oral hypoglycemic agent (Oxramet $5 \mathrm{mg} / 1000 \mathrm{mg}$ OD) with poorly controlled diabetes (HbA1c: $8 \%$ ).

2.2. Differential Diagnoses. In this patient, acute coronary syndrome was most suspected, followed by myocarditis, pericarditis, and stress cardiomyopathy.

2.3. Investigations. The patient's routine blood investigations were sent. An ECG revealed normal sinus rhythm at a rate of 62 beats/min without abnormal QS wave patterns. An echocardiogram revealed normal overall left ventricular function. Troponin I and CPK-MB were normal. The diagnostic CAG revealed a normal LAD and anomalous CX arising from the right coronary cusp along with a CTO in the proximal segment of the anomalous RCA. This RCA was arising from the proximal segment of abnormally originating CX artery.
It also revealed the retrograde filling of distal RCA through grade 2 Werner CCs from the LAD, a long CTO segment with a distal cap at the bifurcation (Figure 1(a)). Therefore, the PCI of an RCA-CTO was scheduled utilizing a primary retrograde strategy.

2.4. Management. Vascular access was obtained using bilateral femoral 7-Fr long sheaths. Particularly, 7-Fr Hyperion AL 1 (Asahi Intecc, Aichi, Japan) and 7-Fr extra backup (EBU) 3.5 (Medtronic, USA) with side holes were utilized to engage the CX arising from the right cusp and the LAD, respectively. A workhorse wire was advanced into the distal abnormal CX. A $3.5 \times 15 \mathrm{~mm}$ noncompliant (NC) balloon (Mozec $^{\mathrm{TM}}$ NC PTCA Balloon) was used to postdilate the previously implanted stent for smooth navigation of gears (Figure 1(b)). A Sion wire (Asahi Intecc, Aichi, Japan) and $150 \mathrm{~cm}$ Corsair ${ }^{\circledR}$ microcatheter (Asahi Intecc, Aichi, Japan) were advanced through the septal CC into the posterior descending artery progressing easily until the distal cap of the occlusion (Figure 1(c)). Similarly, the escalation of the retrograde wire was done. The initial Sion blue was exchanged with Pilot 200 (Abbott Vascular International, Diegem, Belgium), and an attempt to navigate it through the distal cap was unsuccessful. Hence, the distal cap was crossed with a knuckle which could be advanced in subintimal space from distal to proximal RCA allowing delivery of the Corsair $^{\circledR}$ (Figure $1(\mathrm{~d})$ ). Unable to progress further, a 


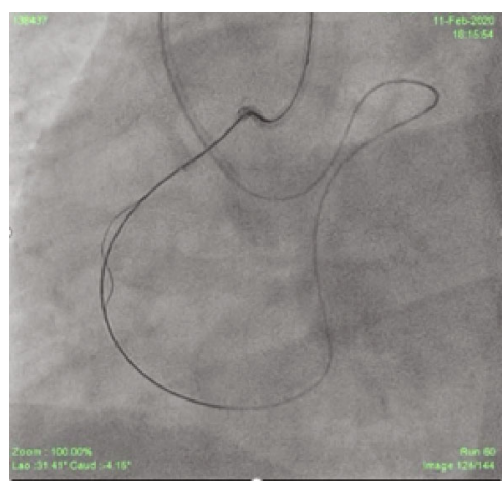

(a)

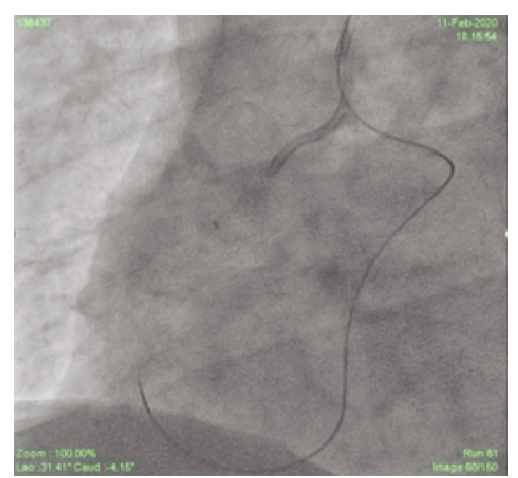

(b)

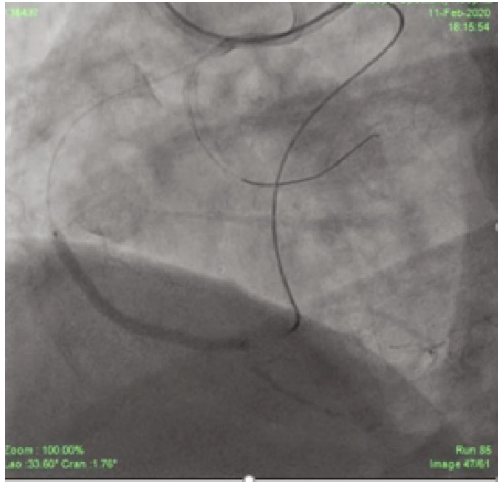

(c)

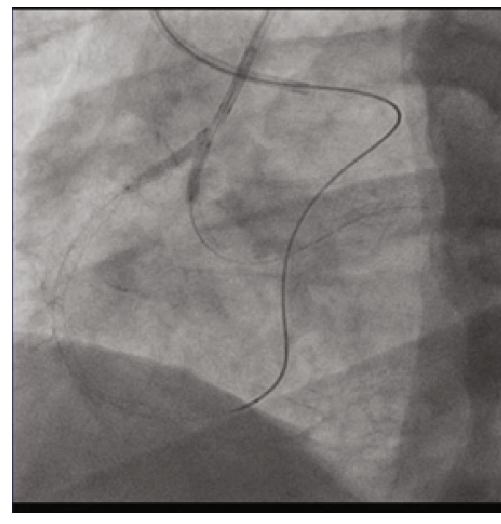

(d)

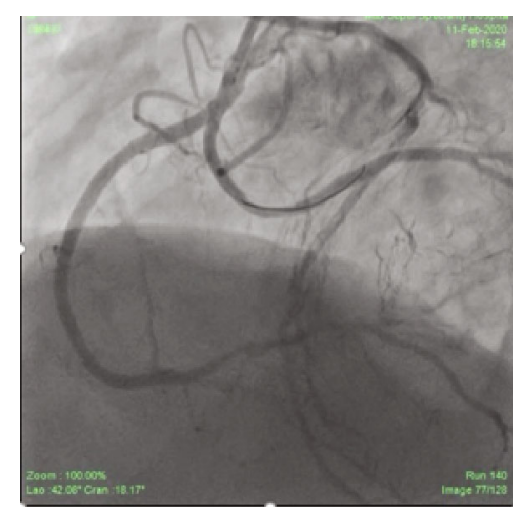

(e)

FIGURE 2: (a) RG3 wire externalized with the help of guideliner. (b) Corsair taken back. (c) 3 stents deployed sequentially. (d).Final proximal kissing. (e) Final result.

decision was made to introduce an anterograde subintimal track through the struts of an already deployed CX stent, from which abnormal RCA was arising, to meet the retrograde wire. A Gaia 2 (Asahi Intecc, Aichi, Japan) was taken through a Finecross microcatheter (Terumo Corp., Tokyo, Japan), escalated to Gaia 3, and finally Conquest Pro 12 (Asahi Intecc, Aichi, Japan). This entered the subintimal space anterogradely but was unable to meet the retrograde wire. To optimize the subintimal space, it was dilated with a $3 \times 15 \mathrm{~mm}$ NC balloon. When this was also futile, a guide extension catheter (Guideliner [Vascular Solutions, Minneapolis, MN, USA]) was positioned in the antegrade subintimal space which created a target lumen enabling rapid reconnection with the true lumen from the retrograde wire (Figure 1(e)). Once the wire engaged the antegrade guide, the procedure became standard reverse CART with RG3 wire (Asahi Intecc, Aichi, Japan) externalized (Figure 2(a)). Following predilation with serial ballooning, 3 overlapping DESs were implanted (Figures 2(b) and 2(c)). The final proximal RCA stent, a $3.5 \times 18 \mathrm{~mm}$ DES (cobalt-chromium everolimus-eluting stent; Xpedition; Abbott Vascular, Santa Clara, California), was deployed with $\mathrm{T}$ and protrusion (TAP) technique touching the CX stent. Final kissing was done with $3 \times 15 \mathrm{~mm}$ NC balloon (Mozec ${ }^{\mathrm{TM}}$ NC PTCA Balloon) in the CX and withdrawn $3.5 \times 18 \mathrm{~mm}$ stent balloon at $12 \mathrm{~atm}$ after the sequential dilatation of respective balloons to 18 atm (Figure 2(d)). The final angiographic result was excellent (Figure 2(e)). Finally, an optical coherence tomography (OCT) run was taken to see the final optimization which showed optimized stent deployment, no edge dissection, good bifurcation angle, and no major complication (Figure 3).

2.5. Follow-Up. The patient was followed up at 3-month intervals in the outpatient department. The patient is free of angina on minimal doses of beta-blocker (Metocard XL $50 \mathrm{mg}$ OD) and long-acting nitrates (Imdur $30 \mathrm{mg}$ OD), and there was no need for sublingual nitrates and calcium channel blockers. He had no symptoms pertaining to cardiovascular symptoms. His ECG was unremarkable, and ECHO showed EF of $60 \%$. Blood sugar was better controlled with $\mathrm{HbAlc}$ of $7 \%$.

\section{Discussion}

The most common cause of failure of PCI for CTO is the inability of wire to cross the occlusion [6,7]. Recently, the retrograde approach through the CCs was introduced to overcome this problem, and several strategies and techniques for the retrograde approach have been developed to increase the efficacy and feasibility of this technique for CTO intervention [8-10]. Recently, Dash [4] described four iterations of reverse CART: (1) conventional reverse CART; (2) contemporary reverse CART involving the use 


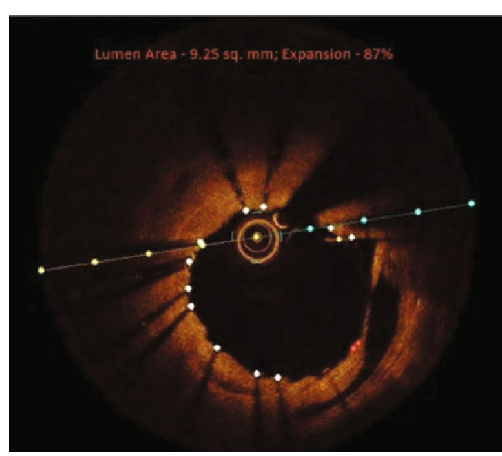

(a)

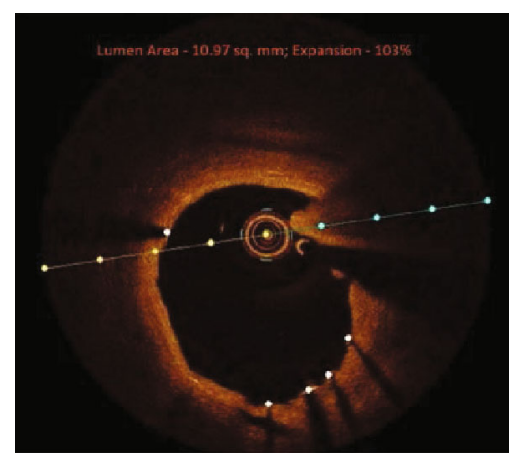

(b)

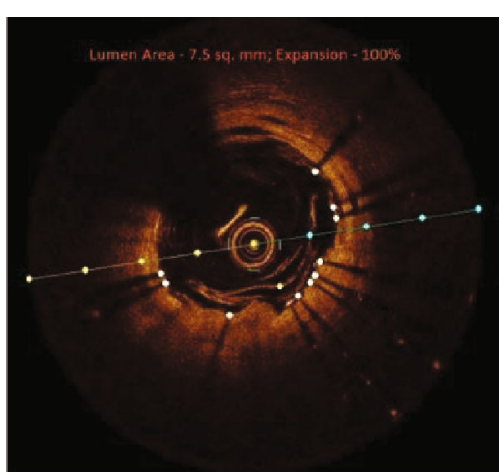

(c)

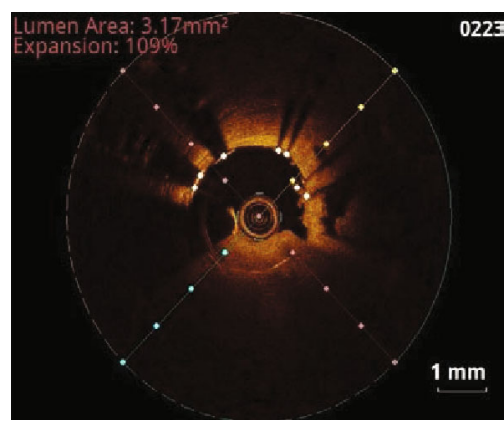

(d)

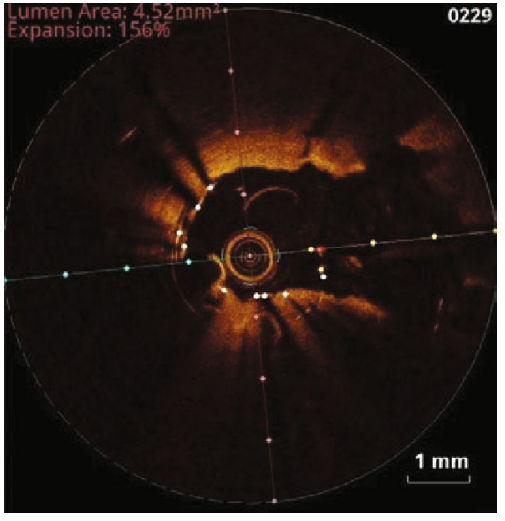

(e)

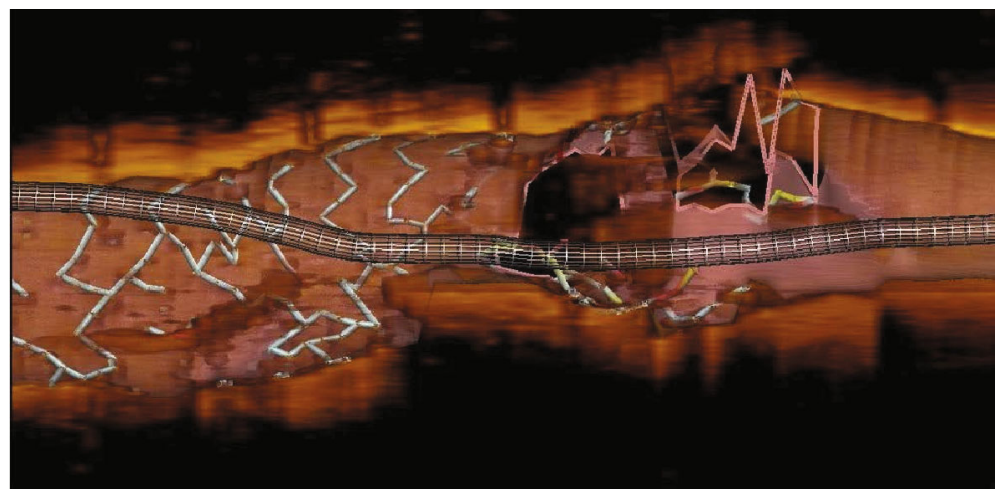

(f)

Figure 3: (a) RCA showing intimal tear within stented segment. (b) Proximal edge with no edge dissection. (c) RCA-distal edge. (d) CX-tissue prolapse. (e) CX bifurcation view-no pinching. (f). CX bifurcation view-longitudinal.

of small antegrade balloons and more active, intentional vessel tracking and penetration; (3) assisted reverse CART involving assistance from intravascular ultrasound (IVUS), stent, guide extension catheter, etc.; and (4) shifting reverse CART. Our case describes how the optimization of the reentry space can be escalated, with the advantage of the guide extensor catheter forming a clear open target, enabling rapid advancement of the retrograde wire into the proximal true lumen. This procedure could have been assisted by IVUS and stent. Stent-facilitated reverse CART is by nature an irreversible procedure as once the stent is deployed, it cannot be removed [4, 11, 12]. Unlike a stent, the guide extension device may be removed or repositioned once there is a failure of connection between the antegrade and retrograde true lumen $[4,11]$. In our case, guideliner-assisted reverse CART was done. Firstly, in our case, the origin of the artery was abnormal; hence, good antegrade preparation was not possible with only AL1 support. Hence, guideliner was used. As the lesion was already stented previously so a good preparation with semicompliant balloon dilatation had to be made to advance guideliner through struts. As we know there may be compression or collapse of subintimal space after antegrade balloon inflation making wiring the true lumen more difficult even if common subintimal space does exist. We overcame this difficulty by the use of mother and child catheter. The advantage of this technique is that we can 
change the position of the guideliner to capture the retrograde microcatheter which otherwise cannot be done if the stent reverse CART technique is used [11]. Also, in our case, it was decided to go retrograde as an initial strategy as there was an abnormal origin and the segment had an overlying stent making antegrade entry ambiguous.

\section{Conclusion}

To conclude, retrograde PCI using reverse CART should be the first-line option for patients of CTO and abnormally originating artery. Also, during reverse CART in these lesions, guideliner can help support antegrade preparation as well as can help in the iteration of reverse CART.

\author{
Abbreviations \\ PCI: Percutaneous intervention \\ CTO: Chronic total occlusion \\ CX: Circumflex \\ CCS: Canadian Cardiovascular Society \\ LAD: Left anterior descending \\ RCA: Right coronary artery \\ CCs: Collateral channels \\ Fr: French \\ AL: Amplatz \\ NC: Noncompliant \\ CART: Controlled antegrade and retrograde tracking \\ CAG: Coronary angiography \\ ECG: Electrocardiogram \\ IVUS: Intravascular ultrasound \\ DAPT: Dual antiplatelet therapy \\ DES: Drug-eluting stent \\ TAP: $\mathrm{T}$ and protrusion \\ OCT: Optical coherence tomography.
}

\section{Data Availability}

The PowerPoint presentation made to support the findings of this study is included within the supplementary information file.

\section{Ethical Approval}

Ethical approval was not required since it is an accepted procedure.

\section{Consent}

Written consent has been obtained to publish the case report from the guardian. The consent copy is available with the authors and ready to be submitted if required.

\section{Conflicts of Interest}

The authors declare that they have no conflicts of interests.

\section{Authors' Contributions}

RM was involved in the collection of data, preparation of the first draft, and revision of the manuscript for important intellectual content. RM, DD, BM, and AS were involved in the collection of data and preparation of the first draft. RM, $\mathrm{BM}$, and AS were involved in the conception of the study, revision of the manuscript for important intellectual content, and approval of the manuscript. All the authors have read and approved the manuscript.

\section{Supplementary Materials}

Link to the PowerPoint presentation of the entire case, including the angiographic and OCT videos, is given in the attached supplementary file. (Supplementary Materials)

\section{References}

[1] R. D. Safian, C. H. McCabe, M. E. Sipperly, R. G. McKay, and D. S. Baim, "Initial success and long-term follow-up of percutaneous transluminal coronary angioplasty in chronic total occlusions versus conventional stenoses," The American Journal of Cardiology, vol. 61, no. 14, pp. 23G-28G, 1988.

[2] C. W. Hamm, W. Kupper, K. H. Kuck, D. Hofmann, and W. Bleifeld, "Recanalization of chronic, totally occluded coronary arteries by new angioplasty systems," The American Journal of Cardiology, vol. 66, no. 20, pp. 1459-1463, 1990.

[3] Y.-H. Kim, S. H. Hwang, C. H. Lim et al., "Reverse controlled antegrade and retrograde subintimal tracking in chronic total occlusion of right coronary artery," Korean Circulation Journal, vol. 42, no. 9, pp. 625-628, 2012.

[4] D. Dash, "Iteration of reverse controlled antegrade and retrograde tracking for coronary chronic total occlusion intervention: a current appraisal," Korean Circulation Journal, vol. 50, no. 10, article e113, pp. 867-879, 2020.

[5] A. M. Mozid, J. R. Davies, and J. C. Spratt, “The utility of a guideliner $^{\mathrm{TM}}$ catheter in retrograde percutaneous coronary intervention of a chronic total occlusion with reverse cart-the "capture" technique," Catheterization and Cardiovascular Interventions, vol. 83, no. 6, pp. 929-932, 2014.

[6] J. Y. Kim, J. Yoon, H. S. Jung et al., "The experience of transradial coronary intervention for chronic total occlusion," Korean Circulation Journal, vol. 33, pp. 805-812, 2003.

[7] T. Noguchi, M. D. Shunichi Miyazaki, I. Morii, S. Daikoku, Y. Goto, and H. Nonogi, "Percutaneous transluminal coronary angioplasty of chronic total occlusions: determinants of primary success and long-term clinical outcome," Catheterization and Cardiovascular Interventions, vol. 49, no. 3, pp. 258-264, 2000.

[8] J. F. Surmely, E. Tsuchikane, O. Katoh et al., "New concept for CTO recanalization using controlled antegrade and retrograde subintimal tracking," The Journal of invasive Cardiology, vol. 18, p. 3348, 2006.

[9] S. Saito, "Different strategies of retrograde approach in coronary angioplasty for chronic total occlusion," Catheterization and Cardiovascular Interventions, vol. 71, no. 1, pp. 8-19, 2008.

[10] N. Kukreja, P. W. Serruys, and G. Sianos, "Retrograde recanalization of chronically occluded coronary arteries: illustration 
and description of the technique," Catheterization and Cardiovascular Interventions, vol. 69, no. 6, pp. 833-841, 2007.

[11] D. Dash, "Retrograde coronary chronic total occlusion intervention using a novel reverse controlled antegrade and retrograde subintimal tracking," Journal of interventional Cardiology., vol. 29, no. 1, pp. 70-74, 2016.

[12] R. Fung, E. B. Wu, and M. H. Jim, "Dislodged stent after stent reverse controlled antegrade and retrograde subintimal tracking (CART) - Is stent reverse CART a necessary tool or an unnecessary evil?," J Cardiol Cases, vol. 16, no. 6, pp. 205209, 2017. 